\title{
Arc-Flash Hazard Calculations in a Electrical Distribution System with Distributed Generation for Electrical Safety Audit
}

\author{
Berkay Gündüz, Burak Dindar, Ömer Gül* \\ Electrical Engineering Department, Istanbul Technical University, Istanbul 34467, Turkey
}

Corresponding Author Email: gulomer@itu.edu.tr

https://doi.org/10.18280/ijsse.110502

Received: 2 July 2021

Accepted: 16 September 2021

\section{Keywords:}

arc flash, distributed generation, electricity distribution, grid topology, PPE, protection coordination

\begin{abstract}
Considering the limited fossil fuel reserves and the damage they cause to the environment, new searches have been made, and distributed generation facilities established in areas close to consumption areas which generate electricity with renewable energy sources have emerged. Connecting distributed generation plants; It has facilitated voltage control, reduced transmission losses and positively affected the supply continuity, however, their effects on short circuit currents, load flows and arc flash energies can cause serious problems if they are not carefully analyzed. In this study, a real distribution feeder system of Boğaziçi Distribution Company has been modeled in ETAP. Arc flash analyzes are carried out according to Institute of Electrical and Electronics Engineers (IEEE) 1584 for the conditions where radial operation, parallel lines and transformers are active in the modeled distribution feeder and the Distributed Generation Plants are connected directly as wind turbine or via inverter as solar panel. Based on the analyzes, it has been observed that the distributed generation plants integrated into the topology cause remarkable increases in arc flash parameters and changes in risk categories. By choosing the wind turbine integration case as an example, overcurrent protection coordination with standard characteristics is provided and differential protection solutions are proposed.
\end{abstract}

\section{INTRODUCTION}

The main purpose of electrical power systems is to meet the electrical energy demands of consumers continuously, with high quality and at an affordable price. Electricity distribution grids are also operated in this direction. However, no matter how careful the design of the electrical distribution grids and the sizing of the devices are, events such as short circuit, arc flash, voltage sags may occur during operation. Protection systems are used to avoid the harmful effects of faults that may occur. The system must be protected with an effective protection system in order to minimize the damage to the responsible personnel, electrical distribution grid and devices in the event of a malfunction. A medium voltage protection system consists of protection relays, breakers, disconnectors, and instrument transformers. There are types of protection relays such as instantaneous overcurrent relays, normal/over reverse time overcurrent relays, directional overcurrent relays, impedance relays and directional impedance relays [1]. IEEE defines relay as an electrical device tasked with detecting the faults and providing the necessary actions in a short time [2]. The protection relays detect the fault as soon as possible, send a trip signal to the breaker of the relevant zone, and separate the faulty zone from the faultless part of the system, thus protecting the system against the negative effects of this fault with minimum power loss. For this reason, the correct sizing of the protection equipment to be used in the electrical grid, their optimum cost and their ability to work in coordination with each other, thus providing the most efficient and safe protection should be the primary target. Parameters to be considered in an effective protection system; selectivity, reliability, backup protection, stability, fast operation and economy [3]. While overcurrent selective protection is provided, the opening time of an output distribution feeder breaker belonging to Turkish Electricity Transmission Corporation (TEIAŞ) is 1.0 seconds at most [4]. The reason why the maximum time determined by TEİAŞ in overcurrent protection is 1.0 seconds is that TEIAŞ wants to prevent the step-down transformers from being damaged and shortening their life as a result of short-circuit faults that occur frequently in Turkish electricity distribution grids. As a solution, the maximum time in the overcurrent selective protection is limited to 1 second until the faults that occur frequently in the distribution grid are reduced with infrastructure improvements. In differential protection, the time required for the protected element to be cleared from a fault, the fault detection time and the trip signal generation time are between 30-40 milliseconds, depending on the operation of the protection algorithm and the transfer of the current value measured at the remote terminal to the local relay. This time provides a much more effective protection compared to overcurrent relays [5].

The decrease in the reserves of fossil fuels and their harmful effects on the environment, the high installation costs of large powerful generation facilities and the widespread use of renewable energy sources with the development of storage technologies have led to the emergence of generation facilities close to consumption areas. Distributed generation facilities are facilities where electricity is generated by using fossil fuels such as steam turbines, natural gas power plants or renewable energy sources such as solar energy and wind energy, located in areas close to the consumption areas of electricity generation [6]. Distributed generation resources can be 
connected to the grid or work as an independent resource in island mode [7]. Distributed generation facilities can produce on a wide scale from a few kilowatts to $300 \mathrm{MW}$ power [8].

Such power plants play an important role in the grid structure, with their ability to be established in a short time, to increase the reliability of the facility they feed due to being close to the load, to reduce losses, to reduce the voltage fluctuation, to reduce carbon emissions, to be sensitive to the environment, to create islands that can generate their own energy with local generation, with their political benefits and increased efficiency [9]. While planning the existing distribution grids, the fact that there will not be any generation in the grid is taken into consideration. The existence of generation sources in the grid depends on the structure of the grid (radial or mesh), the loading capacity of the grid, the diversity of the generation sources and the short circuit power at the connection points; It is expected to affect short-term and stability parameters, voltage drop and rise, power quality (harmonic, flicker, reactive power, voltage and frequency changes), system and transformer grounding, possible ferro resonance events, short circuit events, breaking capacities of equipment [10]. One of the important negative effects of distributed generation facilities is the changes they cause on arc flash energy due to their contribution to short-circuit fault currents. The magnitude of the fault current is affected by the fault location, the location and capacity of the distributed generation plants and loads capacity on each bus [11]. As a result of changes in arc flash energy, it may be necessary to make changes in the arc flash risk category and the Personal Protective Equipment (PPE) that the relevant personnel should use. In order to ensure safe working conditions, these changes need to be analyzed meticulously.

In this study, arc flash analyzes are made in each of the radial, ring feeder topologies and ring feeder topologies with integrated distributed generation plant and these topologies are examined in terms of arc flash energies, arc flash boundaries and hazard categories. As a result of the comparisons, significant increases have been observed in the arc flash parameter values, and changes have been observed in the risk categories of the arc flashes that occurred due to these increases. Appropriate study recommendations are given according to these changing parameters.

\section{ARC EXPLOSION BY FAULT CURRENT}

As a result of short-circuit faults occurring at one or more points of the system, arc flash situations that endanger human health and equipment used are observed. These situations may cause injuries, as well as cause the existing equipment in the system to become unusable or malfunction. In order to avoid these possibilities, while the system is being designed, arc flash analyzes should be done carefully and necessary regulations should be made according to the results obtained. The arc, which is defined as the initiation of current movement between phases or between phase-earth in conductors as a result of perforation of air, was first discovered by Sir Humphry Davy in the early 19th century. It usually starts with the puncture of the insulation between two electrodes that carry an electric current at a certain distance between them. As a result of a high voltage applied, electron transitions between the electrodes begin [12]. Exposure of personnel or equipment to flammable effects as a result of the temperature that can reach four times the surface of the sun as a result of arc flash, cut or burn formation due to molten metal parts scattered around as a result of arc, minor or serious injuries caused by the pressure wave that is released, high sound intensity caused by the explosion Adverse effects such as temporary or permanent hearing loss may be observed due to (up to $140 \mathrm{~dB}$ severity) [13]. Arc faults may occur due to external factors such as dusting, humidity and contact of animals with conductors and bad environmental conditions as well as internal factors such as faulty design of the system, inaccurate operations of switchgears devices and negligence shown in maintenance-repair works [14]

At any point where electric current flows, there exist the risk of an arc flash hazard. Because of this, studies on arc flash have gained importance in recent years. In this direction, a number of methods have been developed for arc flash calculations. NFPA 70E and IEEE 1584 methods which will be detailed in next section are main methods for studying arc flash calculations. Although there are differences between these two methods the main approach to the arc flash analysis can be summarized as follows:

- Collecting system information which are needed for the arc flash calculation

- Setup the system operating configuration

- Calculate 3 phase bolted fault currents

- Calculate arcing fault current (IEEE only)

- Determine arc clearing time (arc duration)

- Determine the incident energy \& Arc flash protection boundary calculations

- Determine hazard/risk category based on NFPA 70E requirements

- $\quad$ Select appropriate PPE equipment's [15].

The increase in arc flash accidents, the start of working at high current values at high voltage levels and increase in shortcircuit fault currents as a result of the increase in the number of consumers can be shown as the reason for this increase in studies. In arc flash analysis, the arc flash energy, operating distance and arc flash boundary, which are released as a result of a short circuit fault, are important parameters. Personnel wearing PPE is shown in Figure 1.

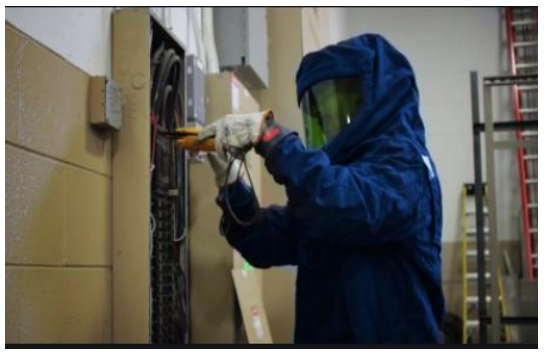

Figure 1. Personnel wearing PPE

\subsection{Arc flash energy}

Arc flash energy is the energy transferred to the unit surface at the operating distance from the arc flash location, its unit is $\mathrm{cal} / \mathrm{cm}^{2}$ or joule $/ \mathrm{cm}^{2}$. At energy levels of $1.2 \mathrm{cal} / \mathrm{cm}^{2}$ and above, second-degree burns can occur on human skin. For comparison, $1 \mathrm{cal} / \mathrm{cm}^{2}$ can be considered equivalent to pressing a lighter into the palm for 1 second [16]. Today, arc flash calculations and procedures are determined by the standards of IEEE and NFPA organizations. In Table 1 the hazard risk category of arc flashes according to NFPA is shown. 
Table 1. NFPA 70E hazard risk category [17]

\begin{tabular}{|c|c|c|}
\hline Hazard Risk Category & Clothing & cal/cm² \\
\hline ( & $\begin{array}{l}\text { Non-melting, flammable materials (untreated cotton, wool, rayon, or silk, or blands of these materials) } \\
\text { with a fabric weight of at least } 4,5 \mathrm{oz} / \mathrm{yd}^{2}\end{array}$ & 0 \\
\hline 1 & FR shirts and FR pants of FR coverall & 4 \\
\hline 2 & Cotton underwear- conventional short sleeve and brief/shorts, plus FR shirts and FR pants & 8 \\
\hline 3 & $\begin{array}{c}\text { Cotton underwear plus FR shirt and FR pants plus FR coverall, or cotton underwear plus two FR } \\
\text { coveralls }\end{array}$ & 25 \\
\hline 4 & Cotton underwear plus FR shirt and FR pants plus multilayer flash suit & 40 \\
\hline Extreme Danger & No PPE available & $>40$ \\
\hline
\end{tabular}

In NFPA 70E Method incident energy is determined as follows.

$$
\begin{gathered}
E_{M A}=5271 D_{A}^{-1.9593} t_{A}\left[0.016 F^{2}-0.0076 F+0.8938\right] \\
E_{M B}=1038.7 D_{B}^{-1.4738} t_{B}\left[0.00093 F^{2}-0.3453 F\right. \\
+5.9675]
\end{gathered}
$$

where, $E_{M A}$ is maximum open air incident energy in cal $/ \mathrm{cm}^{2}$, $E_{M B}$ is maximum cubic box incident energy in $\mathrm{cal} / \mathrm{cm}^{2}, D_{A}$ and $D_{B}$ are distance from arc electrodes in $\mathrm{mm}$ (for $18 \mathrm{in}$. and greater), $t_{A}$ and $t_{B}$ are arc duration in second, $F$ is shortcircuit current in $\mathrm{kA}$ (for the range of $16 \mathrm{kA}$ to $50 \mathrm{kA}$ ).

In IEEE 1584 Method incident energy is determined as follows.

$$
E=C_{f} E_{n}(t / 0.2)\left(610^{x} / D^{x}\right)
$$

where, $E$ is incident energy in $\mathrm{cal} / \mathrm{cm}^{2}, C_{f}$ is calculation factor ( 1.0 for voltages above $1 \mathrm{kV} 1.5$ for voltages below $1 \mathrm{kV}$ ), $E_{n}$ is normalized incident energy in $\mathrm{J} / \mathrm{cm}^{2}, t$ is arc duration in $\mathrm{sec}$, $D$ is distance from the possible arc point to the person in $\mathrm{mm}$ and $x$ is distance exponent [18].

NFPA 70E has a simple application for short-circuit fault currents in the range of $16 \mathrm{kA}-50 \mathrm{kA}$. If the short-circuit fault currents calculated as a result of the analysis are outside the determined limits, the IEEE 1584 method is used. Compared to NFPA 70E, IEEE 1584 allows the determination of arc flash energy and limit values with higher accuracy over a wider range [19]. NFPA 70E allows to determine the limit of arc flash energy, hazard risk category and optimum Arc Flash PPE. Before arc flash analysis, short-circuit analysis and determination of the opening times of the breakers are required.

\subsection{Arc flash boundary and working distance}

The distance between the working personnel and the surface where the arc flash occurs is called the working distance. The working distance is directly proportional to the voltage level. The higher the voltage level, the greater the operating distance. In the NFPA 70E standard, 18 inches for LV switchboards and 36 inches for MV switching equipment are specified. The arc flash boundary (AFB) is defined as the distance at which the arc flash energy shows $1.2 \mathrm{cal} / \mathrm{cm}^{2}$, while other parameters are constant, it increases in direct proportion to the arc flash energy [20].

\section{CASE STUDY}

In this section, the TM 2107 distribution feeder topology belonging to Beyazıt Operations Directorate of Boğaziçi Distribution Company. is modeled with the ETAP software.
On the model, arc flash analyzes were performed according to IEEE 1584 by entering the selective protection parameters of the relays [21]. Finally, the case where a $5 \mathrm{MW}$ wind turbine is directly integrated into the Sultanhamam 2050-2 busbar is selected as an example, and overcurrent protection coordination with International Electrotechnical Commission (IEC) standard characteristics is provided. Differential protection solutions have been proposed for busbars. The feeder topology used in the study is shown in Figure 2. In order to observe the effect of the integration type of distributed generation power plants, analyzes are made by adding solar and wind power plants separately. The solar panel integrated into the system is shown in blue and the wind turbine in red. After examining the effects of the distributed generation power plants integrated into the Sultanhamam 2050- 2 busbar, their number was increased to five in order to observe the changes in the number of these power plants. The busbars to which the distributed generation plants are connected are shown in orange. Finally, switches S1 and S2 are indicated in green.

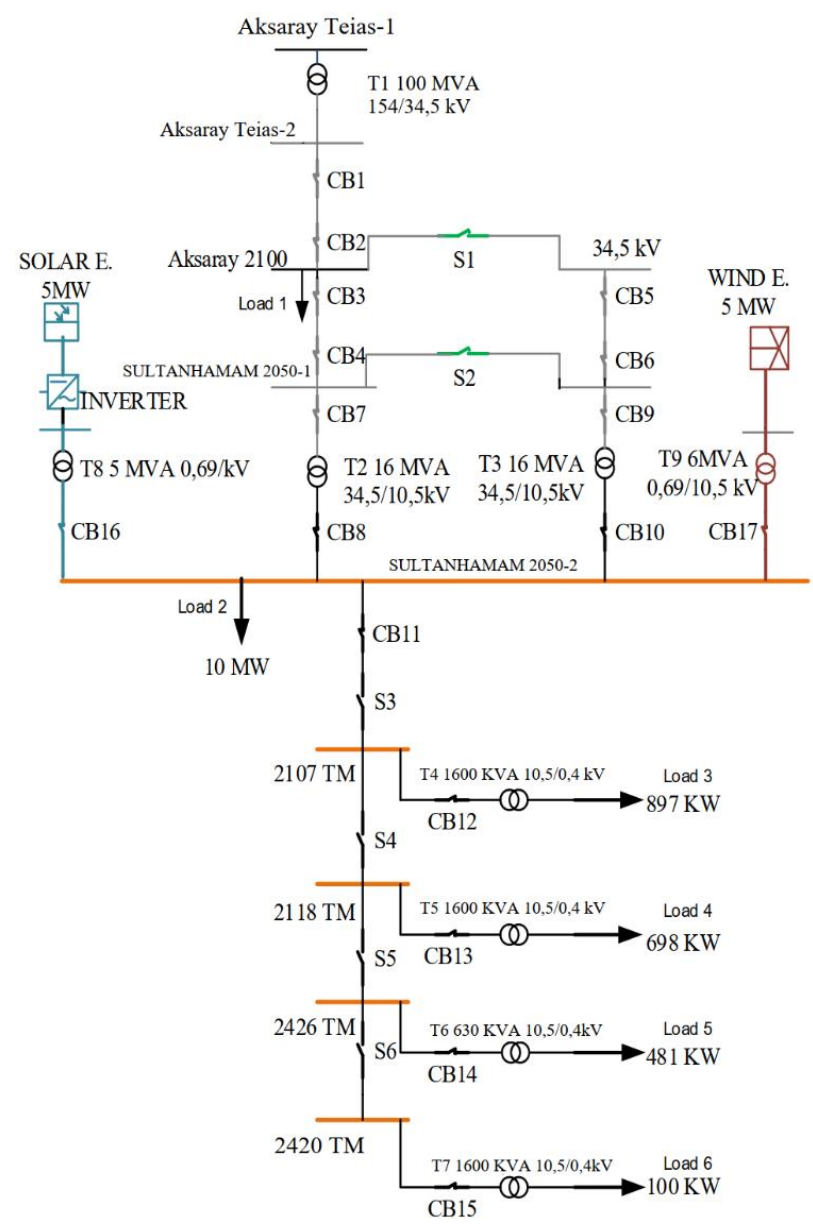

Figure 2. Beyazit TM 2107 
The analyses are examined in different operating conditions of the distribution feeder and in the case of connecting distributed generation power plants. Firstly, the case where the couplings between Aksaray TM 2100 busbar and Sultanhamam 2050-1 busbars in Beyazıt TM 2107 distribution feeder are open, secondly, the operation of the parallel line and transformers and the distribution feeder by closing the open couplings in the previous case, and in the third case, the Sultanhamam 2050-2 busbar is connected to the $5 \mathrm{MW}$ solar panel with an inverter and fourthly, for the case where a $5 \mathrm{MW}$ wind turbine is directly integrated into the Sultanhamam 20502 busbar. Finally, for the cases where distributed generation power plants are integrated, analyzes are carried out by integrating power plants with equal power to the $2107 \mathrm{TM}$, 2118 TM, 2426 TM and 2420 TM busbars, which are medium voltage busbars that the loads are connected, in addition to the Sultanhamam 2050-2 busbar to better observe the effects Selective protection rules are followed while calculating the arc flash energies, the clearing times of the faults and the voltage levels of the busbars in which short circuit occurred are given in the Table 2 .

By integrating distributed generation plants into the grid, changes of arc flash parameters such as magnitude of arc flash energy, distance arc flash boundary and arc flash risk categories that leads to change in PPE, in the system are observed. These changes should be analyzed well and the necessary arrangements for the protection of the relevant personnel and equipment should be provided meticulously as shown as Table 1. In this study, arc flash energy, AFB and NFPA 70E risk categories are given in tables for five different scenarios, and the effects of the integrated distributed generation plants, the type and number of integrations on these parameters are shown. Arc flash parameters are shown in Table 3 when switches A1 and A2 are on and off, without integrating the distributed generation plant into the system.

As can be seen in Table 3, increases in arc flash energies and limits have occurred as a result of the change in the grid operation type. Here, it is necessary to pay particular attention to the $2107 \mathrm{TM}$ and $2118 \mathrm{TM}$ buses. As a result of the increases, the arc flash risk category class in these busbars has changed and the PPE types and features that the personnel in charge must wear have changed. In order to prevent a possible accident, the personnel in charge during the work in these busbars should apply Class 3 risk category protocols.

With switches A1 and A2 closed, a 5 MW solar panel system is integrated via an inverter in the Sultanhamam 20502 busbar in the first case. In the second case, a $5 \mathrm{MW}$ wind turbine is directly integrated. Arc flash parameters for these cases are given in Table 4.

As shown in the Table 4, there has been an increase in arc flash parameters as a result of the distributed generation plants integrated into the system. The point that draws attention here is that the increase caused by the directly connected wind turbine is higher than the solar turbine integrated over the inverter. This is because the inverter limits the short-circuit current since it contains power electronics elements. A particularly striking change occurred at the 2426 TM busbar. In the absence of distributed generation integration, arc flash energy, which is in the $2^{\text {nd }}$ risk category with $7.68 \mathrm{cal} / \mathrm{cm}^{2}$, reaches the level of $7.64 \mathrm{cal} / \mathrm{cm}^{2}$ with a small decrease as a result of the short circuit current limitation of the power electronics elements in the inverter after the solar panel integration. It remained in the risk category, but after the integration of the directly integrated wind panel, it rose to the level of $8.19 \mathrm{cal} / \mathrm{cm}^{2}$ with a critical increase, entering the $3^{\text {rd }}$ risk category and an increase of $10 \mathrm{~cm}$ occurred in the arc flash boundary. As a result of this situation, the PPE that must be used by the personnel working at the busbar and the protocol that must be obeyed have changed.

In the last case, the number of $5 \mathrm{MW}$ solar panel power plants integrated into the system via inverter and wind turbine power plants directly integrated has been increased in order to more clearly observe the effect of distributed generation power plants on the existing arc flash parameters. In addition to Sultanhamam 2050-2 busbar, it has also been added to the 2107 TM, 2118 TM, 2426 TM and 2420 TM busbars. The arc flash parameters observed in these cases are shown in Table 5.

Table 2. Voltage levels and fault clearing times

\begin{tabular}{ccc}
\hline ID & Voltage (kV) & FCT (s) \\
\hline 2107 TM & 10.5 & 0.4 \\
2118 TM & 10.5 & 0.4 \\
2420 TM & 10.5 & 0.4 \\
2426 TM & 10.5 & 0.4 \\
Aksaray 2100 & 34.5 & 1 \\
Aksaray Teiaş-2 & 34.5 & 1 \\
Sultanhamam 2050-1 & 34.5 & 0.8 \\
Sultanhamam 2050-2 & 10.5 & 0.6 \\
Load 3 & 0.4 & 0.2 \\
Load 4 & 0.4 & 0.2 \\
Load 5 & 0.4 & 0.2 \\
Load 6 & 0.4 & 0.2 \\
\hline
\end{tabular}

Table 3. Grid type comparison

\begin{tabular}{|c|c|c|c|c|c|c|}
\hline \multirow[b]{2}{*}{ ID } & \multicolumn{3}{|c|}{ Switches Open } & \multicolumn{3}{|c|}{ Switches Closed } \\
\hline & Total Energy $\left(\mathrm{cal} / \mathrm{cm}^{2}\right)$ & $A F B(m)$ & Risk Cat. & Total Energy $\left(\mathrm{cal} / \mathrm{cm}^{2}\right)$ & $\begin{array}{c}A F B \\
(M)\end{array}$ & Risk Cat \\
\hline $2107 \mathrm{TM}$ & 7 & 1.1 & 2 & 11.29 & 1.4 & 3 \\
\hline $2118 \mathrm{TM}$ & 6.49 & 1.1 & 2 & 9.94 & 1.3 & 3 \\
\hline $2420 \mathrm{TM}$ & 5.31 & 1 & 2 & 7.17 & 1.1 & 2 \\
\hline 2426 TM & 5.55 & 1 & 2 & 7.68 & 1.2 & 2 \\
\hline Aksaray 2100 & 730.75 & 11.3 & Ext. Danger & 730.75 & 11.3 & Ext. Danger \\
\hline Aksaray Teiaş & 730.75 & 11.3 & Ext. Danger & 730.75 & 11.3 & Ext. Danger \\
\hline Sultan hamam $2050-1$ & 519.51 & 9.5 & Ext. Danger & 550.39 & 9.8 & Ext. Danger \\
\hline Sultan hamam 2050-2 & 10.54 & 1.4 & 3 & 17.04 & 1.7 & 3 \\
\hline Load 3 & 11.94 & 1.4 & 3 & 12.61 & 1.5 & 3 \\
\hline Load 4 & 11.82 & 1.4 & 3 & 12.47 & 1.5 & 3 \\
\hline Load 5 & 7.83 & 1.2 & 2 & 8.07 & 1.2 & 3 \\
\hline Load 6 & 11.54 & 1.4 & 3 & 12.15 & 1.5 & 3 \\
\hline
\end{tabular}


Table 4. Comparison of integration form

\begin{tabular}{ccccccc}
\hline \multirow{2}{*}{ ID } & \multicolumn{3}{c}{ Solar Panel } & \multicolumn{3}{c}{ Wind Turbine } \\
\cline { 2 - 7 } 2107 TM & Total Energy $\left({\left.\mathrm{cal} / \mathrm{cm}^{2}\right)}^{2}\right.$ & AFB $(\mathrm{m})$ & Risk Cat. & Total Energy $\left({\left.\mathrm{cal} / \mathrm{cm}^{2}\right)}^{2}\right.$ & AFB $(\mathrm{m})$ & Risk Cat. \\
\cline { 2 - 7 } 2118 TM & 11.31 & 1.4 & 3 & 12.64 & 1.5 & 3 \\
2420 TM & 9.92 & 1.3 & 3 & 10.94 & 1.4 & 3 \\
2426 TM & 7.14 & 1.1 & 2 & 7.61 & 1.3 & 2 \\
Aksaray 2100 & 7.64 & 1.2 & 2 & 8.19 & 1.3 & 3 \\
Aksaray Teiaş & 731.44 & 11.3 & Ext. Danger & 764.16 & 11.6 & Ext danger \\
Sultan hamam 2050-1 & 731.44 & 11.3 & Ext. Danger & 764.16 & 11.6 & Ext. Danger \\
Sultan hamam 2050-2 & 551.08 & 9.8 & Ext. Danger & 577.21 & 10 & Ext. Danger \\
Load 3 & 17.08 & 1.7 & 3 & 19.11 & 1.8 & 3 \\
Load 4 & 12.61 & 1.5 & 3 & 12.74 & 1.5 & 3 \\
Load 5 & 12.47 & 1.5 & 3 & 12.59 & 1.5 & 3 \\
Load 6 & 8.06 & 1.2 & 3 & 8.11 & 1.2 & 3 \\
\hline
\end{tabular}

Table 5. Comparison of the number of distributed generations

\begin{tabular}{ccccccc}
\hline \multirow{2}{*}{ ID } & \multicolumn{3}{c}{ 5x Solar Panel } & \multicolumn{3}{c}{ 5x Wind Turbine } \\
\cline { 2 - 7 } & Total Energy $\left(\mathrm{cal}^{\mathrm{cm}} \mathrm{cm}^{2}\right)$ & $A F B(\mathrm{~m})$ & Risk Cat. & Total Energy $\left({\left.\mathrm{cal} / \mathrm{cm}^{2}\right)}^{A F B}(\mathrm{~m})\right.$ & Risk Cat. \\
\hline 2107 TM & 11.46 & 1.4 & 3 & 17.91 & 1.8 & 3 \\
$2118 \mathrm{TM}$ & 10.16 & 1.3 & 3 & 15.91 & 1.7 & 3 \\
2420 TM & 7.36 & 1.1 & 2 & 10.81 & 1.4 & 3 \\
2426 TM & 7.89 & 1.2 & 2 & 11.79 & 1.4 & 3 \\
Aksaray 2100 & 735.49 & 11.3 & Ext. Danger & 859.92 & 12.3 & Ext. Danger \\
Aksaray Teiaş & 735.49 & 11.3 & Ext. Danger & 859.92 & 12.3 & Ext. Danger \\
Sultan hamam 2050-1 & 554.83 & 9.8 & Ext. Danger & 655.19 & 10.7 & Ext. Danger \\
Sultan hamam 2050-2 & 17.29 & 1.7 & 3 & 26.96 & 2.2 & 4 \\
Load 3 & 12.63 & 1.5 & 3 & 13.06 & 1.5 & 3 \\
Load 4 & 12.51 & 1.5 & 3 & 12.97 & 1.5 & 3 \\
Load 5 & 8.09 & 1.2 & 3 & 8.31 & 1.2 & 3 \\
Load 6 & 12.24 & 1.5 & 3 & 12.67 & 1.5 & 3 \\
\hline
\end{tabular}

Table 6. Differential protection

\begin{tabular}{|c|c|c|c|c|c|c|}
\hline \multirow{2}{*}{ ID } & \multicolumn{3}{|c|}{ Without Diferantial Protection } & \multicolumn{3}{|c|}{ With Diferantial Protection } \\
\hline & Total Energy $\left(\mathrm{cal} / \mathrm{cm}^{2}\right)$ & $A F B(m)$ & Risk Cat. & Total Energy $\left(\mathrm{cal} / \mathrm{cm}^{2}\right)$ & $A F B(m)$ & Risk Cat. \\
\hline Aksaray 2100 & 764.16 & 11.06 & Ext. Danger & 30.57 & 2.3 & 4 \\
\hline Aksaray Teiaş-2 & 764.16 & 11.06 & Ext. Danger & 30.57 & 2.3 & 4 \\
\hline Sultanhamam 2050-1 & 577.21 & 10 & Ext. Danger & 28.86 & 2.2 & 4 \\
\hline
\end{tabular}

As can be seen in Table 5, as a result of the increase in distributed generation plants integrated into the system, the arc flash energy in the $3^{\text {rd }}$ Risk category of $17.04 \mathrm{cal} / \mathrm{cm}^{2}$ in Sultanhamam 2050-2 busbar showed a small increase as a result of solar panel integration, and $17.29 \mathrm{cal} / \mathrm{cm}^{2}$ level, but during the wind turbine integration this increase was much higher and increased to the $4^{\text {th }}$ Risk category with 26.96 $\mathrm{cal} / \mathrm{cm}^{2}$. The energy that rose to $19.11 \mathrm{cal} / \mathrm{cm}^{2}$ as a result of one wind turbine integration increased to $26.96 \mathrm{cal} / \mathrm{cm}^{2}$ in the case of 5 wind turbines. In addition, the arc flash boundary, which increased by $10 \mathrm{~cm}$ as a result of the integration of one power plant, increased by $50 \mathrm{~cm}$.

IEC Standard Inverse selective protection coordination study has been carried out for the case where one wind turbine is integrated into the system over the Sultanhamam 2050-2 busbar. For example, the reason for choosing this situation is that in the case of direct integration, the effect of the distributed generation plant on the system parameters is more effective and observable, since there is no power electronic system in between. The coordination curves of the selective overcurrent protection system in the event of a short-circuit fault occurring in the Load 6 busbar, which is determined as an example in the scenario mentioned, are given in Figure 3.
As can be seen from the analysis, the arc flash energy in the busbars close to the source is at very high values due to increased voltage level and decreased impedance value that leads higher short circuit current. When differential protection is provided in Aksaray Teiaş-2, Aksaray 2100 and Sultanhamam 2050-1 busbars close to the source, where the arc flash energy is very high, the fault clearing times of these busbars will be 0.04 seconds for a fault condition. In the case of differential protection, arc flash parameter values that do not allow operation are greatly reduced to below $40 \mathrm{cal} / \mathrm{cm}^{2}$, which can be operated without cutting the power. The arc flash parameters observed after differential protection are given in Table 6 .

As shwon in Table 6, very high arc flash energies in Aksaray Teiaş-2, Aksaray 2100 and Sultanhamam 2050-1 busbars have decreased to levels where operation can be performed without power interruption, if these busbars are protected with differential relays, by following appropriate PPE and procedures. Especially in the busbars close to the source, with the differential protection solutions, the possibility of operating without the energy interruption, the maximum protection of the personnel and equipment can be realized. 


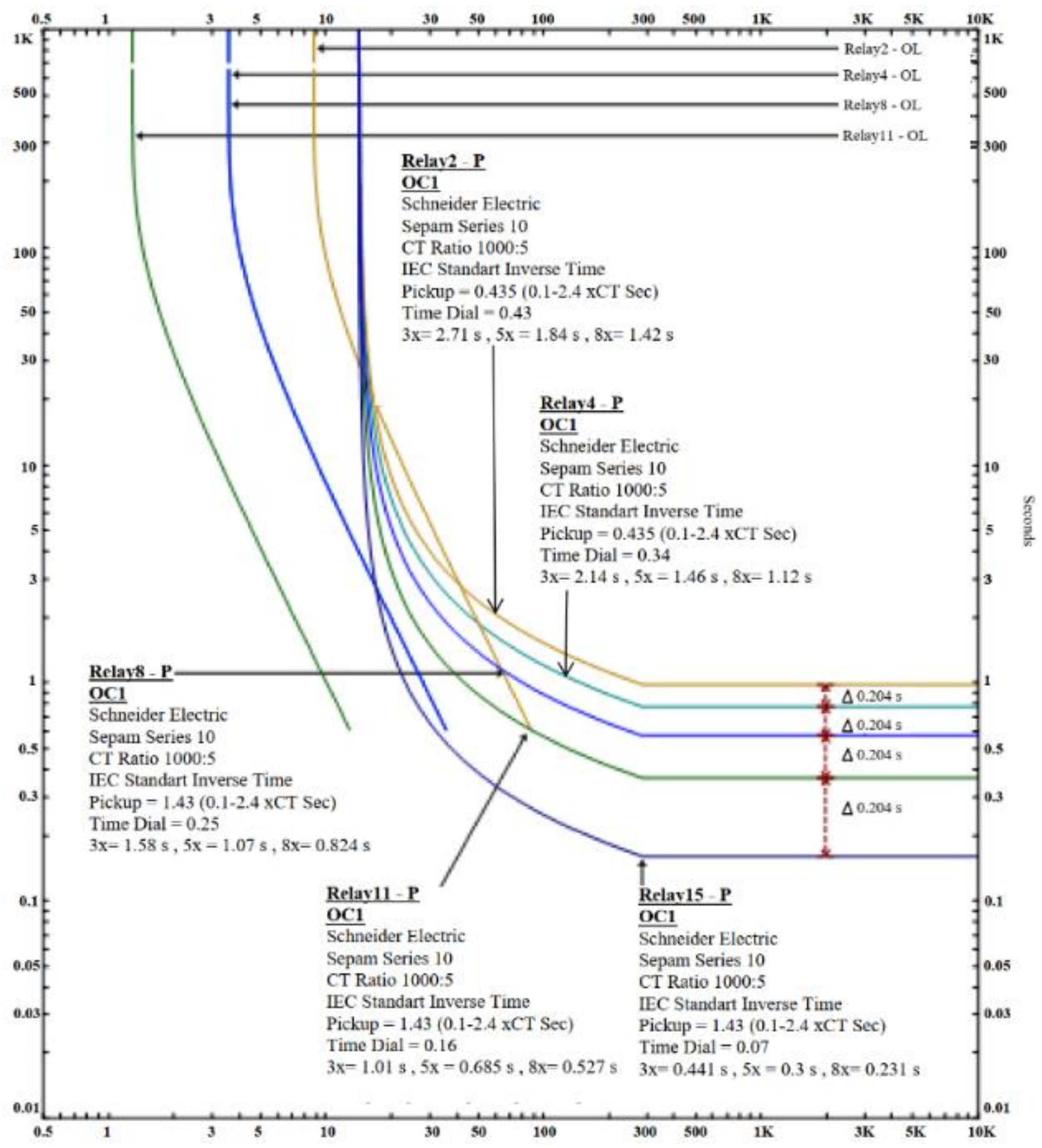

Figure 3. Overcurrent selective protection curves

\section{CONCLUSION}

The effects of the electricity generation facilities integrated into the system should be analyzed in order to prevent the system or its equipment from being permanently damaged in the face of failures in the electrical power systems and to ensure the safety of human health at the best level. The primary aim of this study is to determine the negative effects of the distributed generation facilities integrated into the system and the connection types of these facilities on arc flash parameters in electricity distribution grids and to offer differential protection solutions to limit these effects. As a result of changes in arc energy, problems such as the need to develop personal protective equipment that should be used, and the life safety of employees may be endangered.
In this study, a real system is modeled with the ETAP software. Arc flash analyzes on the modeled sample system are given in tables. As a result of the analysis, besides the many positive effects of distributed generation plants such as reducing current losses, correcting voltage, reducing carbon emissions, being sensitive to the environment and creating islands that can produce their own energy with local generation, attention is drawn to their negative effects on arc flash parameters. The sample system initially focused on the effects of grid type without distributed generation. In this case, since the impedance value seen at the fault points decreased with the effect of parallel lines and transformers, the amplitude values of the short circuit currents increased compared to the fault current amplitudes in radial operating conditions. Then, in case of parallel lines and transformers, distributed 
generation plants are added, and arc flash analyzes are made again.

In the analyzes for the cases which the distributed generation plants are directly connected to the Sultanhamam 2050-2 busbar and with an inverter, it has been observed that the directly connected wind turbine increases the amplitude of the arc flash energy more than the solar panel connected with the inverter. It is observed that the number of power plants increased significantly with the increase in the number of power plants. The reason for this is the current limitation of the power electronics elements in the inverter. Arc flash energy has also increased with the increasing short circuit current, and changes that may occur in the selection of important protection elements such as safe working distance and PPE used should be considered in cases where distributed generation plants are in the system. Changes observed in arc flash energies, arc flash boundaries and risk categories with the connection of distributed generation plants or the change of the operating topology of the electrical power system should be considered and adverse scenarios that may occur should be prevented. In order to fulfill this requirement, the PPE equipment to be used in different operating conditions and the safe working distances must be regulated. Closer to the source, the impedance value decreases, so the short-circuit current and arc flash energy reach very high values. During live maintenance operations, since there is no suitable PPE for energies of $40 \mathrm{cal} / \mathrm{cm}^{2}$ and above, it is very dangerous to work at this level, and PPEs that should be used between $0 \mathrm{cal} / \mathrm{cm}^{2}$ and $40 \mathrm{cal} / \mathrm{cm}^{2}$ energy levels and working procedures change after certain values.

While the changes in voltage levels and short-circuit currents that will occur in case of adding a new source to the grid in the projects are considered, yet the changes in the arc flash parameters are not considered sufficiently. In this study, it has been showed that the parameters of the arc flash such as energy levels and PPE that should be used changed significantly. Accordingly, decision makers need to consider the effects of new sources to be added to the grid on arc flash parameters. In order to prevent serious accidents that may occur, it is necessary to determine the risk categories meticulously by performing arc flash analysis, to use special personal protective equipment for this situation and to follow the operation procedures. For live maintenance operations, it is possible to clear the fault as soon as possible, to limit the arc flash energy to occur and to provide suitable working conditions, by using differential protection relays during operation, in to ensure the most active protection, especially in the works carried out in the busbars close to the source.

\section{REFERENCES}

[1] Cheung, H., Hamlyn, A., Wang, L., Yang, C., Cheung, R (2009). Investigations of impacts of distributed generations on feeder protections. 2009 IEEE Power \& Energy Society General Meeting, Calgary, AB, Canada, pp. 1-7. https://doi.org/10.1109/PES.2009.5275220

[2] Farzin, H., Monadi, M., Fotuhi-Firuzabad, M., Savaghebi, M. (2021). A reliability model for overcurrent relays considering harmonic-related malfunctions. International Journal of Electrical Power \& Energy $\quad$ Systems, 131: 107093. https://doi.org/10.1016/j.ijepes.2021.107093

[3] Monadi, M., Zamani, M.A., Candela, J.I., Luna, A.,
Rodriguez, P. (2015). Protection of AC and DC distribution systems Embedding distributed energy resources: A comparative review and analysis. Renewable and Sustainable Energy Reviews, 51: 15781593. https://doi.org/10.1016/j.rser.2015.07.013

[4] Electricity Grid Regulation, Turkish EMRA Standard, 2014.

[5] Zhou, C., Zou, G., Zang, L., Du, X. (2021). Current differential protection for active distribution networks based on improved fault data self synchronization method. IEEE Transactions on Smart Grid. https://doi.org/10.1109/TSG.2021.3116608

[6] Dulău, L.I., Abrudean, M., Bică, D. (2014). Effects of distributed generation on electric power systems. Procedia Technology, 12: 681-686. https://doi.org/10.1016/j.protcy.2013.12.549

[7] Palizban, O., Kauhaniemi, K. (2015). Hierarchical control structure in microgrids with distributed generation: Island and grid-connected mode. Renewable and Sustainable Energy Reviews, 44: 797-813. https://doi.org/10.1016/j.rser.2015.01.008

[8] Gullapelli, M.M. (2021). A review on need of integrated distributed energy sources with seamless mode transition in perspective of controlling, protection, equilibrium and island operation of microgrids. Journal of Advanced Research in Electrical Engineering and Technology, 3(1): 1-6.

[9] Deng, J., Wang, Y., Sun, D., Zheng, C., Yan, H., Gao, X. (2021). Research on the effect of IIDG on local feeder protection. Power Generation Technology, 42(1): 115121. https://doi.org/10.12096/j.2096-4528.pgt.19001

[10] Barker, P.P., De Mello, R.W. (2000). Determining the impact of distributed generation on power systems. I. Radial distribution systems. 2000 Power Engineering Society Summer Meeting (Cat. No. 00CH37134), Seattle, WA, USA, pp. 1645-1656. https://doi.org/10.1109/PESS.2000.868775

[11] Gumilar, L., Habibi, M.A., Sholeh, M., Nugroho, W.S. (2020). Analysis of short circuit on four types wind power plants as distributed generation. 2020 International Conference on Smart Technology and Applications (ICoSTA), Surabaya, Indonesia, pp. 1-5. https://doi.org/10.1109/ICoSTA48221.2020.157059908 9

[12] Das, J.C. (2020). Arc Flash Hazard Analysis and Mitigation. John Wiley \& Sons.

[13] Bhatia, S. (2020). Electrical safety-arc flash hazard analysis. Journal International Association on Electricity Generation, Transmission and Distribution, 33(2): 19-24.

[14] Nowak, K., Janiszewski, J., Dombek, G. (2021). The possibilities to reduce arc flash exposure with arc fault $\begin{array}{lll}\text { eliminators. } & \text { Energies, } & 14(7) \text { : }\end{array}$ https://doi.org/10.3390/en14071927

[15] Rubini, B., Krishnakumar, R. (2020). A review on ARC flash analysis and calculation methods. 2020 Fourth International Conference on Computing Methodologies and Communication (ICCMC), pp. 975-979. https://doi.org/10.1109/ICCMC48092.2020.ICCMC000181

[16] Floyd, H.L. (2014). Facilitating application of electrical safety best practices to "other" workers. IEEE Transactions on Industry Applications, 51(2): 1348-1352. https://doi.org/10.1109/TIA.2014.2358848

[17] Clark, C.G., Engineer, G.S. (2016). "The Basics of Arc 
Flash" GE Industrial Solutions web site accessed on Oct, 5.

[18] Valdes, M.E., Floyd, H.L. (2021). Considerations for adapting IEEE 1584-2002 arc flash study results to a post IEEE 1584-2018 risk assessment. IEEE Transactions on Industry Applications https://doi.org/10.1109/TIA.2021.3105620

[19] Gopila, M. (2021). Arc flash analysis based on IEEE 1584-2018 and NFPA70E-2018. Turkish Journal of Computer and Mathematics Education (TURCOMAT), 12(9):

2869-2873 https://doi.org/10.17762/turcomat.v12i9.4373

[20] Reeves, A., Freyenberger, M., Hodder, M. (2020). Understanding the effect of electrode configuration on incident energy and Arc-flash boundary. IEEE Transactions on Industry Applications, 56(6): 6069-6075. https://doi.org/10.1109/TIA.2020.3023918

[21] Sezgin, M.S. (2015). Impacts of hidden failures on protection coordination in electrical distribution systems with distributed generation, MSc Thesis, Istanbul Technical University.

\section{GLOSSARY}

AFB Arc flash boundary

IEEE Institute of Electrical and Electronics Engineers

IEC International Electrotechnical Commission

NFPA National Fire Protection Association

PPE Personal Protective Equipment

TEIASŞ Turkish Electricity Transmission Corporation

\section{NOMENCLATURE}

$C_{f}$

D

E

$E_{M A}$

$E_{M B}$

$E_{n}$

$F$

$t$

$x$ calculation factor

distance from arc electrodes, $\mathrm{mm}$ incident energy, $\mathrm{cal} / \mathrm{cm}^{2}$ maximum open air incident energy, $\mathrm{cal} / \mathrm{cm}^{2}$ maximum cubic box incident energy, $\mathrm{cal} / \mathrm{cm}^{2}$ normalized incident energy, $\mathrm{J} / \mathrm{cm}^{2}$ short-circuit current, $\mathrm{kA}$ arc duration, $\mathrm{s}$ distance exponent 